$$
\begin{gathered}
\text { WoRKING PAPERS } \\
\text { No. } 1 / 2013(36) \\
\text { MICHAt BRZEZIŃSKI } \\
\text { ASYMPTOTIC AND BOOTSTRAP } \\
\text { INFERENCE FOR TOP } \\
\text { INCOME SHARES }
\end{gathered}
$$




\title{
Asymptotic and bootstrap inference for top income shares
}

\author{
Michat BRZEZIŃSKI \\ Faculty of Economic Sciences \\ University of Warsaw \\ e-mail: mbrzezinski@wne.uw.edu.pl
}

\begin{abstract}
We analyse statistical inference for top income shares in finite samples. The asymptotic inference performs poorly even in large samples. The standard bootstrap tests give some improvement, but can be unreliable. Semi-parametric bootstrap approach is accurate in moderate and larger samples.
\end{abstract}

Keywords:

top income shares, income distribution, inference, bootstrap, semi-parametric bootstrap

JEL:

$\mathrm{C} 15, \mathrm{C} 14, \mathrm{I3}$ 


\section{Introduction}

In recent years, there has been growing interest in economic analysis of top income shares, that is, the shares of total income held by the richest $x \%$ (e.g., $10 \%, 5 \%$ or $1 \%$ ) of the population. Long-run time series of top income shares for more than twenty countries have been constructed (Atkinson and Piketty 2007, 2010; Atkinson et al. 2011), which facilitates the study of trends in income distributions that spans decades and, in some cases, the entire 20th century. Although much of this literature uses income tax data, several contributions are also based on household survey data. The latter are used to estimate top income shares when tax return data are not available or only available for a limited time span (see, e.g., Leigh and Van der Eng 2009; Piketty and Qian 2009) or when one wants to compare results from tax returns and survey data (Burkhauser et al. 2012).

Income tax data used in the recent literature on top income shares are usually based on very large samples, sometimes covering the whole population of taxpayers. On the other hand, household survey data often come from samples of moderate or rather small size. In this context, the question of sampling variability of top income shares calculated using survey data arises naturally. Although asymptotic inference methods for income shares exist since 1980s (Beach and Davidson 1983), their performance in finite samples has not been studied. Moreover, as shown recently by Davidson and Flachaire (2007) asymptotic and standard bootstrap inferences for some popular inequality measures such as the Theil index are unreliable even in very large samples. ${ }^{1}$ They argued that the reason for the poor performance of asymptotic and standard bootstrap methods is the sensitivity of some inequality indices to the exact nature of the upper tail of the income distribution. Since top income shares are constructed as ratios of (portions of) upper tails to total income of a population, it may be

\footnotetext{
${ }^{1}$ See also Cowell and Flachaire (2007), Davidson (2012) and Schluter (2012) for other contributions to this literature.
} 
expected that the problems observed by Davidson and Flachaire (2007) are equally or even more relevant for top income shares.

This paper uses Monte Carlo simulations to study the finite-sample performance of asymptotic inference for top income shares. We also compare the asymptotic inference with the standard bootstrap and a non-standard semi-parametric bootstrap method proposed by Davidson and Flachaire (2007). We find that the asymptotic approach fails in providing accurate inference, while the performance of standard bootstrap is better but not satisfactory. Monte Carlo results suggest that the semi-parametric bootstrap performs well in moderate samples.

\section{Asymptotic variance estimation for top income shares}

The asymptotic approach to variance estimation for quantile shares was introduced by Beach and Davidson (1983), who derived the distribution-free asymptotic joint variance-covariance structure for income quantile shares and Lorenz curve ordinates (cumulative income shares). ${ }^{2}$ Let $X=\left(x_{1}, \ldots x_{N}\right)$ be an income distribution with the cumulative distribution function (CDF) $F$, mean income $\mu$ and variance $\lambda^{2}$. An income quantile of order $p$, $\xi_{p}$, with $0<p<1$, is defined implicitly by $F\left(\xi_{p}\right)=p$. Quantiles divide the sample into $n=1 / p$ quantile groups; $p_{i}$ $=i / n, i=1, \ldots, n-1$, denote the quantile proportions. In the case of deciles, there are $K=9$ quantiles with quantile proportions $p_{1}=0.1, \ldots, p_{9}=0.9$. The top quantile share is defined as

$$
\psi_{T} \equiv 1-\Phi\left(\xi_{K}\right)
$$

where $\Phi\left(\xi_{K}\right)$ is a population Lorenz curve ordinate for income quantile $K$, defined as

\footnotetext{
${ }^{2}$ See also Beach and Kaliski (1986) for inference for income shares with sample weights. Binder and Kovačević (1995) and Verma and Betti (2011) use other approaches to derive asymptotic inference for income shares.
} 


$$
\Phi\left(\xi_{K}\right)=\frac{1}{\mu} \int_{0}^{\xi_{K}} x d F(x)=p_{K} \frac{\gamma_{K}}{\mu}
$$

where $\gamma_{K}$ is the conditional mean of incomes less than or equal to $\xi_{K}$. The top income share (1) can be estimated using a plug-in estimator as

$$
\hat{\psi}_{T}=1-p_{K} \frac{\hat{\gamma}_{K}}{\hat{\mu}}
$$

Distribution-free asymptotic variance of top quantile shares is derived by Beach and Davidson (1983) as

$$
\begin{aligned}
\operatorname{Var}\left(\psi_{T}\right) & =\frac{1}{N} \frac{p_{K}}{\mu^{2}}\left[\lambda^{2} \frac{p_{K} \gamma_{K}^{2}}{\mu^{2}}+\lambda_{K}^{2}\left(1-\frac{p_{K} \gamma_{K}}{\mu}\right)+\left(1-p_{K}\right)\left(\xi_{K}-\gamma_{K}\right)^{2}\right. \\
& \left.-2 \frac{p_{K} \gamma_{K}}{\mu}\left(\xi_{K}-\gamma_{K}\right)\left(\mu-\gamma_{K}\right)\right]
\end{aligned}
$$

where $\lambda_{K}^{2}$ is the conditional variance of incomes less than or equal to $\xi_{K}$. It can be estimated by replacing $\mu, \gamma_{K}, \lambda^{2}, \lambda_{K}^{2}$, and $\xi_{K}$ with their sample estimates.

\section{Asymptotic and bootstrap tests}

In order to test a hypothesis that $\psi_{T}=\psi_{T}^{0}$, for some value of $\psi_{T}^{0}$, we can use estimate of the top income share, $\hat{\psi}_{T}$, given by (3) and estimate of its variance, $\widehat{\operatorname{Var}}\left(\hat{\psi}_{T}\right)$ from (4) and construct the following asymptotic $t$-type statistic

$$
W=\frac{\widehat{\psi}_{T}-\psi_{T}^{0}}{\left[\widehat{\operatorname{Var}}\left(\hat{\psi}_{T}\right)\right]^{0.5}} .
$$

In our simulations, we use data drawn from the 4-parameter Generalized Beta of the Second Kind (GB2) distribution, which is widely used in modelling earnings and income distributions and known to fit these distributions very well (McDonald 1984, Kleiber and Kotz 2003, Dastrup et al. 2007). See Kleiber and Kotz (2003) for a detailed review of the GB2 properties. 
The sample sizes in our simulations are $n=500,1000,2000,3000,4000,5000$, and $10000 .{ }^{3}$ We use the GB2 parameter estimates from McDonald and Ransom (2008), who have shown that the GB2 distribution it the best choice for fitting 2000 family income data for the US. For our choice of parameters, the "true" values of the top $10 \%, 5 \%$ and $1 \%$ income shares that we analyse in our simulations, are $0.345895,0.231211$ and 0.088844 , respectively. We calculate an asymptotic $P$ value from (5) replacing $\psi_{T}^{0}$ by an appropriate "true" value and using the Student distribution with $n$ degrees of freedom.

We compare the performance of asymptotic inference with standard bootstrap method known as the percentile- $t$ or bootstrap- $t$ method. The test is constructed as follows. First, we compute $W$ statistics as given by (5) from the original sample and then we draw $B$ bootstrap samples of the same size as the original sample. We set $B$ to 199 in our simulation. For each bootstrap sample $j, j=1, \ldots, 199$, we compute $W_{j}^{*}$ statistic in the same way as $W$ was computed from the original sample, but with $\psi_{T}^{0}$ replaced by the index $\hat{\psi}_{T}$ estimated from the original sample. The bootstrap $P$ value is the proportion of the bootstrap samples for which the bootstrap statistic $W_{j}^{*}$ is more extreme than $W$.

We complement the asymptotic and standard bootstrap tests with a non-standard bootstrap test proposed by Davidson and Flachaire (2007) in the context of improving inference for the Theil index of inequality. Their semi-parametric bootstrap test combines parametric modelling of the upper tail with the standard non-parametric bootstrap for the rest of the distribution. The test is implemented as follows. First, the $W$ statistic as defined in (5) is computed for the original sample of size $n$. Second, a Pareto distribution is fitted to the $c$ largest incomes with $c$ chosen using a procedure introduced by Dupuis and Victoria-Feser (2006). Third, the "true" value of a top quantile share, $\psi_{T}^{*}$, is computed for a semi-parametric

\footnotetext{
${ }^{3}$ We do not provide results for smaller sample sizes because when $n<500$ the number of observations needed to calculate the top incomes, especially belonging to the top $1 \%$, is too small to allow for a meaningful variance estimation of $\psi_{T}$.
} 
distribution consisting of the estimated Pareto tail and the sample values for the rest of the distribution. Fourth, a bootstrap sample is drawn with probability $p_{t}=c / n$ from the Pareto distribution and with probability $1-p_{t}$ from the $n\left(1-p_{t}\right)$ smallest observations from the original sample. Fifth, for the bootstrap sample the bootstrap statistic $W^{*}$ is computed using (5) with $\psi_{T}^{0}$ replaced by $\psi_{T}^{*}$. The last two steps of this procedure are repeated 199 times. The semi-parametric bootstrap $P$ value is the proportion of $W_{j}^{*}, j=1, \ldots, 199$, that are more extreme than $W$.

\section{Simulation results}

The results of our simulations in terms of errors in rejection probability (ERPs), that is, the difference between the actual and nominal probabilities of rejection (set to 5\%), are shown in Figures 1-3. A test with no size distortion would produce a plot coinciding with the horizontal axis. The number of Monte Carlo replications is 10,000. From Figure 1 we see that the ERPs for the asymptotic test are large in small and moderate samples and, unsurprisingly, the largest for the top $1 \%$ income share, which is affected most by the extremely large income observations. The ERPs are slowly decreasing as the sample size increases, but even in large samples the distortion is unacceptable. For example, the ERP for the top $1 \%$ income share and $n=10,000$ is 0.0542 , which means that the actual rejection probability is $10.42 \%$ when the nominal level is 5\%. The ERPs for other top income shares are smaller, but still significant; they are 0.0242 and 0.0167 for the top $5 \%$ and the top $10 \%$ shares, respectively. These results suggest that the asymptotic inference for top income shares is unreliable in finite samples.

Figure 2 shows results for the standard bootstrap tests. We can see that the ERPs are reduced for all top income shares. However, the ERP of the top 1\% share is still as much as 
0.0305 even when $n=10,000$. The convergence of the ERPs to 0 for other top income shares is slow.

Figure 1. ERPs for asymptotic tests

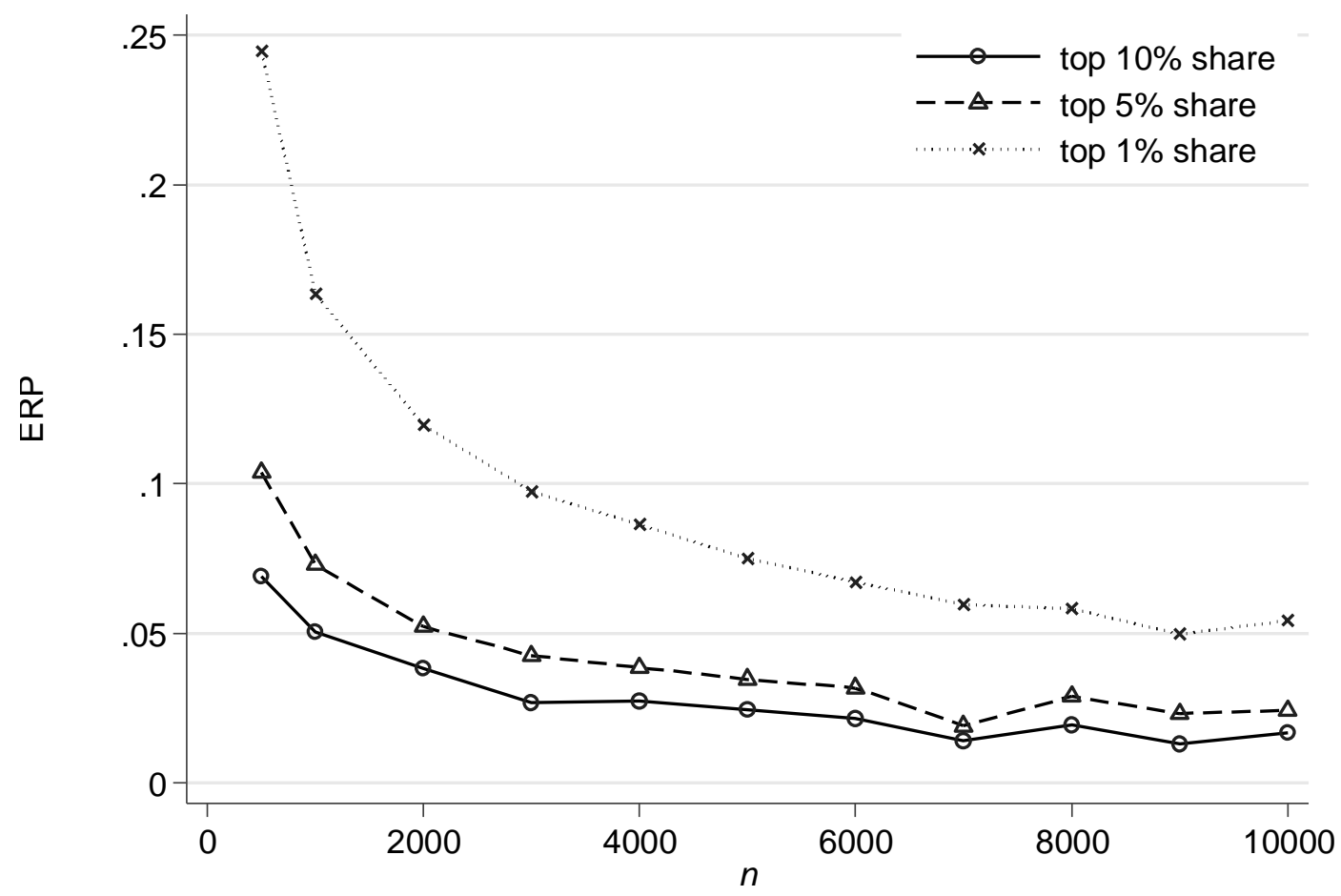

Figure 2. ERPs for standard bootstrap tests

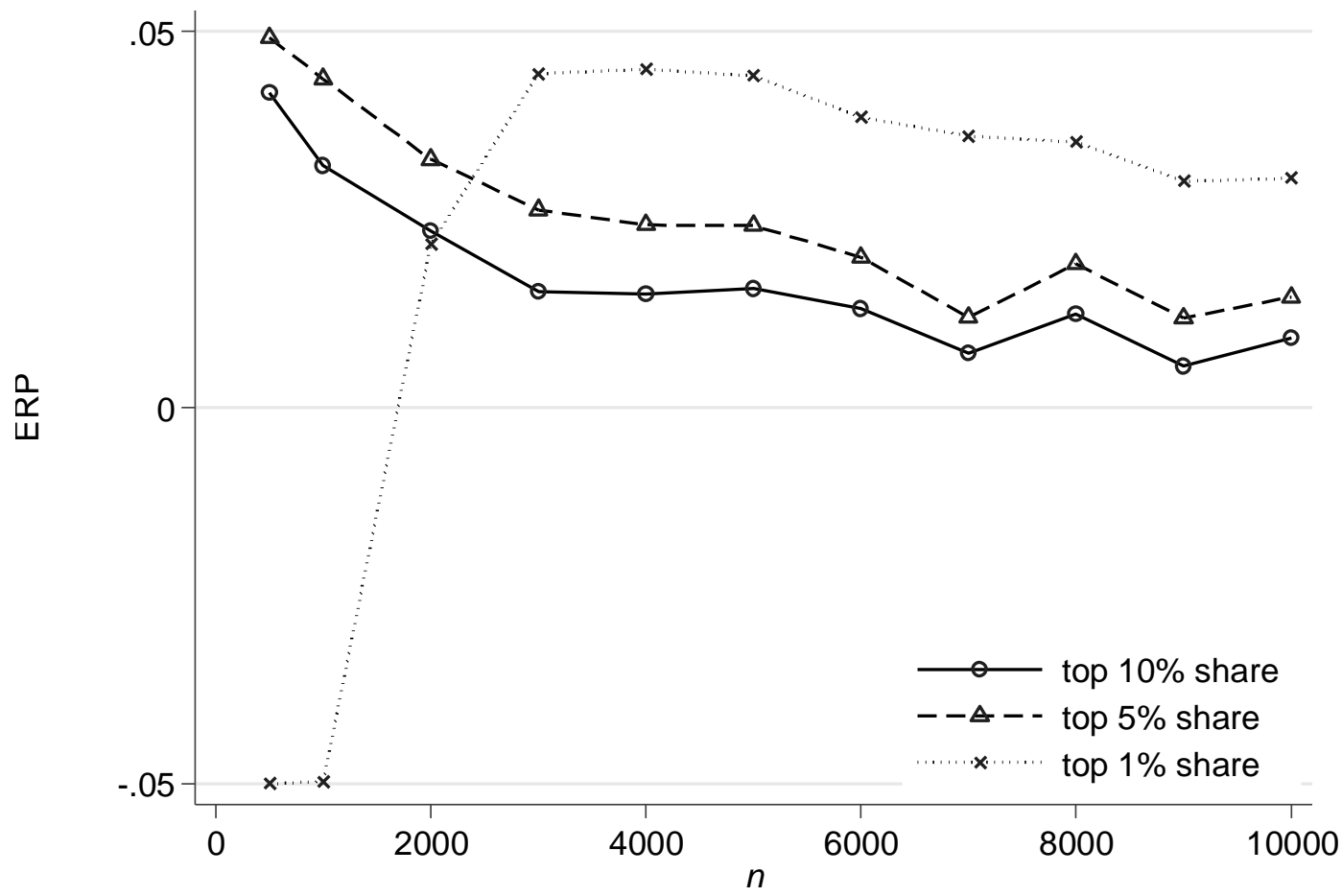


They remain above $2 \%$ in moderate samples and fall below $1.5 \%$ only for bigger samples $(n \geq$ $7,000)$. The bootstrap test for the top $1 \%$ share underrejects when the sample size is small $(n \leq$ 1,000). Overall, we conclude that the standard bootstrap inference for top income shares, and especially for the top $1 \%$ share, can be unreliable even in large samples.

Figure 3. ERPs for semi-parametric bootstrap tests

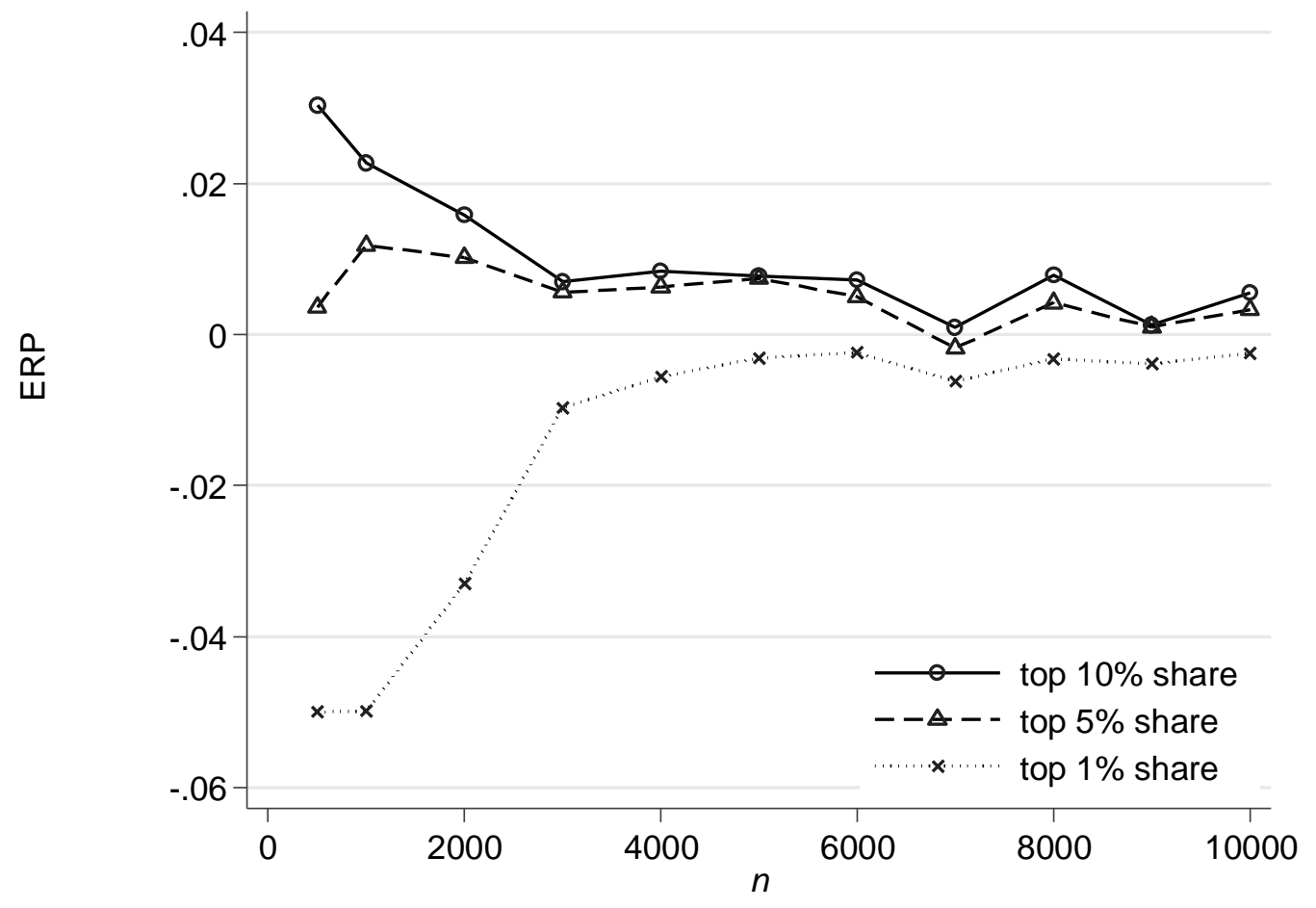

Finally, the ERPs for the semi-parametric bootstrap tests are given in Figure 3. Comparing with results for the standard bootstrap, we see that the ERPs are further reduced. The ERPs for all top income shares fall below $\pm 1 \%$ already for $n=3,000$. We conclude that the semi-parametric bootstrap tests provide accurate inference for the standard top income shares in moderate samples. 


\section{Conclusions}

This paper has used Monte Carlo simulations to study the finite sample performance of asymptotic and bootstrap inference for top income shares. We have found that the asymptotic inference performs poorly even in large samples. The standard bootstrap tests provide some improvement, but can be unreliable, especially in case of the top $1 \%$ share. Semi-parametric bootstrap tests give accurate inference for moderate and larger samples. The practical recommendation is that using the asymptotic approach for variance estimation of top income shares, as implemented for example in Jenkins (2006), can lead to serious errors.

\section{References}

Atkinson, A. B., T. Piketty, and E. Saez (2011) "Top Incomes in the Long Run of History," Journal of Economic Literature, Vol. 49, No. 1, pp. 3-71.

Atkinson, A.B. and T. Piketty (2007) Top Incomes over the Twentieth Century: A Contrast between Continental European and English-Speaking Countries (Volume 1), Oxford: Oxford University Press.

Atkinson, A.B. and T. Piketty (2010) Top Incomes over the Twentieth Century: A Global Perspective (Volume 2), Oxford: Oxford University Press.

Atkinson, A.B., T. Piketty, \& E. Saez (2011). Top incomes in the long run of history. Journal of Economic Literature, 49(1), 3-71.

Beach, C. M., \& Davidson, R. (1983). Distribution-free statistical inference with Lorenz curves and income shares. The Review of Economic Studies, 50(4), 723-735.

Beach, C. M., \& Kaliski, S. F. (1986). Lorenz curve inference with sample weights: an application to the distribution of unemployment experience. Applied Statistics, 35(1), 38-45.

Binder, D. A., \& Kovacevic, M. S. (1995). Estimating some measures of income inequality from survey data: an application of the estimating equations approach. Survey Methodology, 21, 137-146.

Burkhauser, R.V., S. Feng, S. Jenkins, \& J. Larrimore (2012). Recent trends in top income shares in the USA: reconciling estimates from March CPS and IRS tax return data. Review of Economics and Statistics, 94(2), 371-388. 
Cowell, F. A., \& Flachaire, E. (2007). Income distribution and inequality measurement: the problem of extreme values. Journal of Econometrics, 141(2), 1044-1072.

Dastrup, S., Hartshorn, R. \& McDonald, J. (2007). The impact of taxes and transfer payments on the distribution of income: A parametric comparison. Journal of Economic Inequality, 5(3), 353-369.

Davidson, R. (2012). Statistical inference in the presence of heavy tails. The Econometrics Journal, 15(1), C31-C53.

Davidson, R., \& Flachaire, E. (2007). Asymptotic and bootstrap inference for inequality and poverty measures. Journal of Econometrics, 141(1), 141-166.

Dupuis, D.J. \& Victoria-Feser, M.-P. (2006). A robust prediction error criterion for Pareto modelling of upper tails. The Canadian Journal of Statistics, 34(4), 639-658.

Jenkins, S. P. (2006). svylorenz: Stata module to derive distribution-free variance estimates from complex survey data, of quantile group shares of a total, cumulative quantile group shares, SSC Archive S456602, http://ideas.repec.org/c/boc/bocode/s456602.html.

Kleiber, C., \& Kotz, S. (2003). Statistical size distributions in economics and actuarial sciences (Vol. 381). Wiley-Interscience.

Leigh, A. \& P. Van der Eng (2009). Inequality in Indonesia: What can we learn from top incomes? Journal of Public Economics, 93(1-2), 209-212.

McDonald, J. B. (1984). Some Generalized Functions for the Size Distribution of Income. Econometrica, 52(3), 647-663.

McDonald, J. B. \& Ransom, M. (2008). The Generalized Beta Distribution as a Model for the Distribution of Income: Estimation of Related Measures of Inequality." In D. Chotikapanich (ed.), Modeling Income Distributions and Lorenz Curves, Springer: New York, 147-166.

Piketty, T. \& N. Qian (2009). Income Inequality and Progressive Income Taxation in China and India, 1986-2015. American Economic Journal: Applied Economics, Vol. 1, No. 2, pp. 53-63.

Schluter, C. (2012). On the problem of inference for inequality measures for heavy-tailed distributions. The Econometrics Journal, 15(1), 125-153.

Verma, V. \& Betti, G. (2011). Taylor linearization sampling errors and design effects for poverty measures and other complex statistics. Journal of Applied Statistics, 38(8), 15491576. 


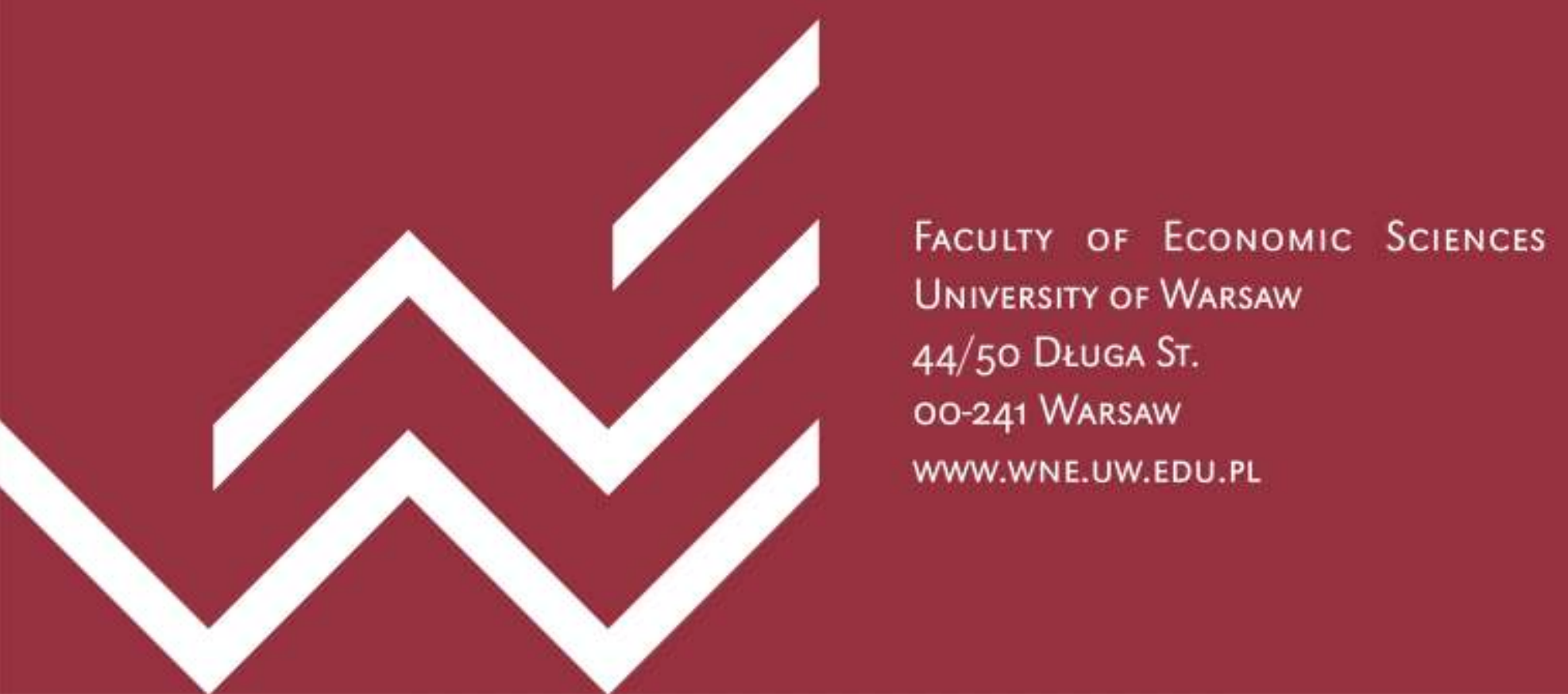

